\title{
Non-perturbative Effects Near the Upper Critical Field in Type-II Superconductors
}

\author{
Safi R. Bahcall \\ Department of Physics, University of California, Berkeley CA 94720
}

\begin{abstract}
The properties of a conventional type-II superconductor near the upper critical field are usually calculated using a perturbative expansion in the strength of the order parameter. Here we show that perturbation theory breaks down near the upper critical field and that this breakdown leads to unusual behavior near the phase transition.
\end{abstract}

Standard microscopic calculations of the properties of type-II superconductors near the upper critical field rely on two assumptions: 1) a perturbative expansion in the strength of the superconducting order parameter and 2) weak magnetic field. These calculations yield an upper critical field $H_{c 2}(T)$ close to what is observed experimentally [1.2]. More complete solutions to the Gor'kov equations, which do not rely on the weak magnetic field assumption, yield low temperature divergences in $H_{c 2}(T)$ [3] which have not been observed. In this paper we show that the origin of these divergences is the breakdown of perturbation theory, and that the full, nonperturbative solution to the Gor'kov equations describes tails of residual superconductivity above $H_{c 2}$.

The microscopic description of type II superconductors is based on Gor'kov's extension of the BCS theory

$$
\mathcal{H}=\int d \mathbf{r} c_{\mathbf{r} \sigma}^{\dagger} \mathcal{H}_{0}(\mathbf{r}) c_{\mathbf{r} \sigma}-\frac{1}{2} V_{0} \Omega \int d \mathbf{r} c_{\mathbf{r} \sigma}^{\dagger} c_{\mathbf{r} \sigma^{\prime}}^{\dagger} c_{\mathbf{r} \sigma^{\prime}} c_{\mathbf{r} \sigma},
$$

which describes a short-range attractive ineraction of strength $V_{0}>0$ for particles near the Fermi energy, $\left|E-E_{F}\right|<E_{c}$. $\mathcal{H}_{0}$ is the bare Hamiltonian $\mathcal{H}_{0} \equiv(1 / 2 m)$ $(i \nabla-e \mathbf{A} / c)^{2}-E_{F}, c_{\mathbf{r} \sigma}^{\dagger}$ are the electron creation operators, and $\Omega$ is the system volume. The variational approach to this problem is to solve for the eigenstates of the two-body Hamiltonian

$$
\mathcal{H}^{\prime}=\int d \mathbf{r} \Psi_{\mathbf{r}}^{\dagger}\left[\begin{array}{cc}
\mathcal{H}_{0}(\mathbf{r}) & \Phi(\mathbf{r}) \\
\Phi^{*}(\mathbf{r}) & -\mathcal{H}_{0}^{*}(\mathbf{r})
\end{array}\right] \Psi_{\mathbf{r}}
$$

where $\Psi_{\mathbf{r}}^{\dagger} \equiv\left[c_{\mathbf{r} \uparrow}^{\dagger} c_{\mathbf{r} \downarrow}\right]$. Minimizing $\langle\mathcal{H}\rangle$ leads to the selfconsistency condition

$$
\Phi(\mathbf{r})=-V_{0} \Omega\left\langle c_{\mathbf{r} \uparrow} c_{\mathbf{r} \downarrow}\right\rangle .
$$

The standard result for the upper critical field $H_{c 2}$ follows from evaulating Eq. (3) perturbatively in $\Phi(\mathbf{r})$. Both sides of this equation are linear to lowest order so setting the resulting coefficients equal gives the condition which determines $H_{c 2}$. Although this is usually done using Green's functions, here we consider the wavefunctions directly.

First we separate the magnitude of the order parameter

$$
\Phi(\mathbf{r}) \equiv \phi f(\mathbf{r})
$$

where $\phi$ is real and positive and $f(\mathbf{r})$ is normalized so that $\frac{1}{\Omega} \int|f(\mathbf{r})|^{2} d \mathbf{r}=1$. Next, we write the eigenstates of the bare Hamiltonian as

$$
\mathcal{H}_{0} \psi_{\alpha}(\mathbf{r})=\varepsilon_{\alpha} \psi_{\alpha}(\mathbf{r})
$$

and define new quasiparticle operators by rotating to this basis: $d_{\alpha \sigma}^{\dagger}=\int d \mathbf{r} \psi_{\alpha}(\mathbf{r}) c_{\mathbf{r} \sigma}^{\dagger}$. We can then define a pairing matrix

$$
A_{\alpha \beta} \equiv \int d \mathbf{r} f(\mathbf{r}) \psi_{\alpha}(\mathbf{r}) \psi_{\beta}(\mathbf{r}),
$$

so that the variational two-body Hamiltonian is

$$
\mathcal{H}^{\prime}=\Psi^{\dagger}\left[\begin{array}{cc}
\varepsilon & \phi A \\
\phi^{*} A^{\dagger} & -\varepsilon
\end{array}\right] \Psi
$$

where $\Psi$ is the vector of quasiparticle operators $\Psi^{\dagger} \equiv$ $\left[\cdots d_{\alpha \uparrow}^{\dagger} \cdots \cdots d_{\beta \downarrow} \cdots\right]$ and $\varepsilon$ is the diagonal matrix of bare eigenvalues $\left|\varepsilon_{\alpha}\right|<E_{c}$.

Eq. (7) is the general form of the pairing Hamiltonian that occurs when there is no translation or time-reversal symmetry restricting the states which can be paired. If $\phi$ is small and we can use perturbation theory, the variational energy difference between the superconducting and normal state, $\langle\mathcal{H}\rangle_{S}-\langle\mathcal{H}\rangle_{N}$, that follows from Eqs. (3) and (7) is, keeping the lowest two orders in $\phi$ :

$$
\Delta E_{\text {pert }}(H)=\phi^{2}\left(\frac{1}{V_{0}}-\sum_{\alpha \beta} \frac{\left|A_{\alpha \beta}\right|^{2}}{\left|\varepsilon_{\alpha}+\varepsilon_{\beta}\right|}\right)+b \phi^{4} .
$$

This describes a continuous phase transition at the field $H$ at which the quantity in parenthesis vanishes. When the electron gas wavefunctions are used, this yields the semiclassical result for $H_{c 2}$ plus some cutoff-dependent corrections [6].

Non-perturbative effects are illustrated most simply for a two-dimensional electron gas at zero temperature. Bare energies are Landau levels, multiples of the cyclotron energy $\hbar \omega_{H}$. The Fermi energy is at the highest occupied Landau level, so the matrix $\varepsilon$ in Eq. (7) is

$$
\varepsilon=\hbar \omega_{H}\left(\begin{array}{lllllll}
\ddots & & & & & & \\
& -2 & & & & & \\
& -1 & & & & \\
& & 0 & & & \\
& & & & 2 & \\
& & & & & \ddots
\end{array}\right)
$$


Near the phase transition $\phi$ can be taken to be small compared to $\hbar \omega_{H}$, but before perturbation theory can be applied degenerate levels must be diagonalized. The pairing splits each Landau level into two with energies $\varepsilon_{m} \pm \phi\left|A_{m, 2 N_{f}-m}\right|$. For the filled levels below the Fermi energy the number of occupied states shifted up in energy equals the number shifted down in energy so there is no net contribution to the energy from this splitting. When the level right at the Fermi energy is partially filled, however, more states will be shifted down in energy (Fig. 11). This will contribute a term linear in $\phi$ to the energy difference between the superconducting and normal state, which then has the form:

$$
\Delta E_{2 \mathrm{D}}(H)=-\epsilon \phi+a(H) \phi^{2}+O\left(\phi^{3}\right) .
$$

In the relevant weak field limit, $E_{F} \gg \hbar \omega_{H}$, and assuming the lowest Landau level component for the order parameter, a calculation for the electron gas yields $\epsilon=\alpha \rho_{0} \hbar \omega_{H}$ and $a(H)=\rho_{0} \ln \left(H / H_{c 2}^{0}\right)$, where $\rho_{0}$ is the zero-field density of states at the Fermi energy and $H_{c 2}^{0}$ is the perturbative result for the upper critical field. Here $\alpha \approx 0.67 \nu / N_{f}^{1 / 4}$ where $\nu$ is the fraction of the highest Landau level which is filled (or one minus this fraction if it is more than half-filled) and $N_{f}$ is the total number of filled levels $N_{f}=E_{F} / \hbar \omega_{H}=\left(k_{F} \ell_{H}\right)^{2} / 2 \sim 10^{3}-10^{4}$ for typical materials.

Minimizing Eq. (10) for the energy difference yields the result shown in Fig. 2: the order parameter develops a "tail" of persisting superconductivity. In the limit of small $\epsilon$, the magnitude of the order parameter in the tail is $\phi=\epsilon / 2 a(H)$. Since $\epsilon$ depends on the filling factor of the highest level, $\phi$ oscillates with magnetic field at a frequency $\delta H / H_{c 2} \approx 1 / N_{f}$ about the upper dashed line in Fig. 2.

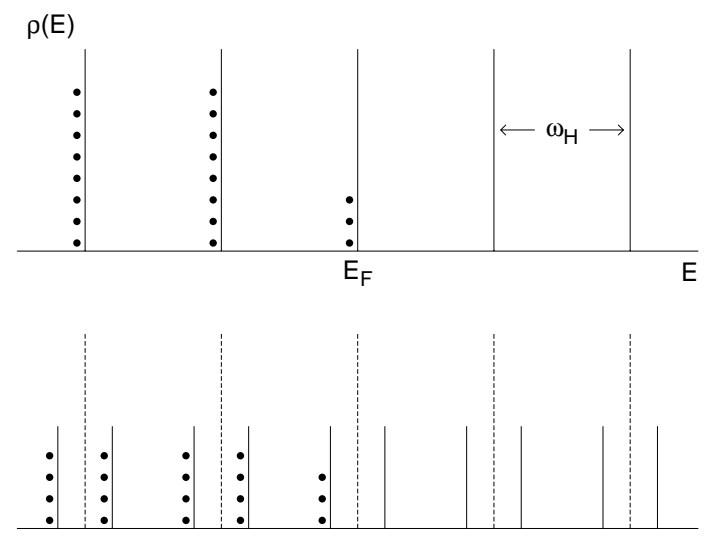

FIG. 1. Top: Density of states of an ideal two-dimensional electron gas. At zero temperature the Landau level at the Fermi energy is partially filled. Bottom: The pairing splits each level into two levels separated by an energy $\propto \phi$, the magnitude of the order parameter. Electrons in the partially filled level contribute a net $O(\phi)$ term to the energy.
This form of residual superconductivity depends on the exact degeneracy of the Landau level poles of a noninteracting two-dimensional electron gas in a magnetic field. In real materials interactions will broaden these levels. When the broadening is larger than $\sim \epsilon / a(H)$, there will no longer be a term linear in $\phi$ in the energy difference and this effect will be suppressed.

It is believed that the effect of impurities in two dimensions at zero temperature is to cause extended states to appear only at discrete energies, as evidenced by the quantum Hall effect [7, 8]. To the extent to which the most relevant interactions are those between the extended states, pairing will split these levels as in Fig. 11 and cause the two-dimensional residual superconductivity to occur in superconductors at temperatures low enough and materials clean enough that the quantum Hall effect could be resolved in the normal state. Such conditions have not yet been achieved in thin metallic films, but may eventually be.

Although the two-dimensional form of residual superconductivity depends on large degeneracies in the spectrum, a weaker form occurs more generally, when the normal-state spectrum is flat. To see where this comes from, consider first the zero-field BCS theory. The difference between the ground state energy of the superconductor and of the normal state can be written, for a uniform gap $\Delta$,

$$
\Delta E_{B C S}=\frac{\Delta^{2}}{V_{0}}-2 \rho_{0} \int_{0}^{E_{c}} d E\left(\sqrt{E^{2}+\Delta^{2}}-E\right) .
$$

The first term is from the expectation value of the interaction term in $H$ and the second is the shift in the electronic energy levels. The normal state spectrum is assumed flat in the range $E_{c}$ around the Fermi energy.

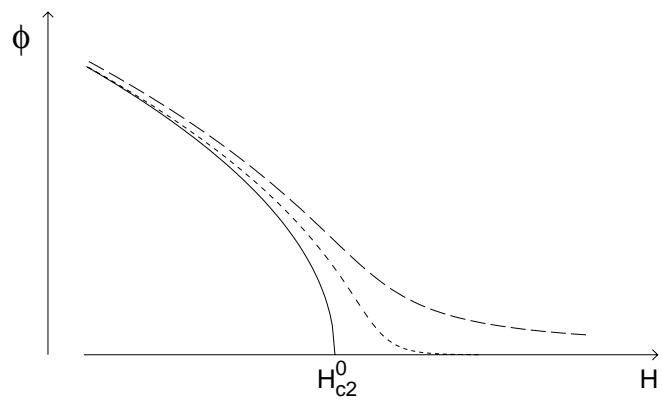

FIG. 2. Solid line is the standard perturbative result for the order parameter as a function of field near the transition: $\phi \propto\left(H_{c 2}-H\right)^{1 / 2}$. Two-dimensional residual superconductivity at $T=0$, Eq. (10), yields rapid oscillations about the top dashed line; the three-dimensional form, Eq. (12), yields the middle dashed line. 
Even for small $\Delta$ compared to $E_{c}$, perturbation theory can not be used to evaluate the integral in the second term of Eq. (11). The exact answer is $\frac{1}{2} \Delta^{2}\left[\ln \left(2 E_{c} / \Delta\right)+\right.$ $\left.\frac{1}{2}+O\left(\Delta^{2} / E_{c}^{2}\right)\right]$ : the $\Delta^{2} \ln \Delta$ term is non-perturbative. In the BCS case this term exactly cancels the $\Delta^{2} / V_{0}$ term, leaving the net condensation energy $\frac{1}{2} \rho_{0} \Delta^{2}$. In a magnetic field, the balance between the $O\left(\phi^{2}\right)$ terms determines the perturbative upper critical field, as in Eq. (8), and a net $\phi^{2} \ln \phi$ term remains:

$$
\Delta E_{3 \mathrm{D}}(H)=\epsilon \phi^{2} \ln \phi+a(H) \phi^{2}+O\left(\phi^{3}\right) .
$$

This form for the energy difference can be derived for the three-dimensional electron gas in the formal limit, as before, $\phi \ll \hbar \omega_{H}$. The additional complication is that the bare Hamiltonian does not have the simple structure of Eq. (9); the energies are offset by an amount $\delta_{z}$ which depends on the $z$-momentum: $\varepsilon_{n}\left(k_{z}\right)=\hbar \omega_{H}\left(n+\frac{1}{2}\right)+$ $k_{z}^{2} / 2 m-E_{F} \equiv \hbar \omega_{H}\left(n-N_{f}+\delta_{z}\right)$. Integrating with respect to $\delta_{z}$ those levels within $\hbar \omega_{H}$ of the Fermi energy gives an integral of the form which occurs in Eq. (11). The result is Eq. (12) with $\epsilon \approx 0.22 \rho_{0} / N_{f}^{1 / 2}$ and $a(H)=$ $\rho_{0} \ln \left(H / H_{c 2}^{0}\right)$.

The form of Eq. (12) is not sensitive to the assumption of a non-interacting electron gas in a magnetic field. If we suppose that 1) the spectrum in the normal state is flat, and 2) the radiply varying matrix elements that enter Eq. (77) are random, we obtain the results shown in Fig. 3. The diagonal matrix $\varepsilon$ is taken from a uniform distrubition $-N / 2<\varepsilon_{i}<N / 2$, corresponding to the flat normal state spectrum. The $N \times N$ matrix $A_{\alpha \beta}$ is taken to be a complex symmetric matrix normalized to $\operatorname{Tr} A^{\dagger} A=N$ with individual elements Gaussian distributed. (The $\phi \rightarrow 0$ results are not very sensitive to this choice of distribution, or to a more realistic approximation where $A_{\alpha \beta}$ falls off exponentially with $\left|\varepsilon_{\alpha}-\varepsilon_{\beta}\right|$.) We calculate the shift in the ground state energy $\Delta E$, divide by $\phi^{2}$, and examine the limit as $\phi \rightarrow 0$. If the result were purely perturbative, corresponding to Eq. (8), we would see a constant limit. Instead, a logarithmic upturn is clearly visible, corresponding to Eq. (12).

Whether the non-perturbative effects will be visible in a given material depends on the number of states $N$ that are mixed by the pairing. In the electron gas case, the symmetry of the vortex lattice generates a conserved momentum and $N \sim \sqrt{N_{f}}$. The effects of disorder in a superconductor, when time-reversal symmetry has been explicitly broken by the presence of a magnetic field, are not well understood. (The semiclassical AbrikosovGor'kov description is not sufficient to address the effects discussed here.) If the number of states being paired becomes macroscopic then non-perturbative effects will be suppressed. If the effective number of states being paired remains small, the number of states inside a volume determined by the scattering length rather than the coherence length, then non-perturbative effects will remain and be enhanced.

A further interesting possibiltity is that the random matrix model described above is a useful model for the spectrum of a granular superconductor [9] in a field, where pairing takes place within a grain and is in the mesoscopic regime. In that case $N$ is the number of states in a grain being paired, and can be as low as $10^{2}$.

At fields far above the conventional $H_{c 2}$, minimizing Eq. (12) for the order parameter strength gives $\phi \sim \exp (-a(H) / \epsilon)$. The order parameter becomes exponentially suppressed, as shown in Fig. 2. When $\phi$ is sufficiently small the superconductivity will become disfavored due to normal-state Pauli paramagnetism or pair-breaking effects, such as magnetic impurities, which are not included in the idealized Hamiltonian in Eq. (1). It is an interesting possiblity, however, that more exotic order parameter configurations [10] would allow the residual state to persist even in the presence of such effects.

At finite temperature, the quantity to be minimized is the Gibbs free energy. As in the BCS case, the energy difference $\Delta E(T)=\phi^{2} / V_{0}-2 \int d E E \delta \rho(E) \tanh (E / 2 T)$, where $\delta \rho$ is the change in the density of states, and the entropy is given by the Fermi gas expression. The factor of $\tanh (E / 2 T)$ cuts off the $\phi^{2} / E^{2}$ rise of $\delta \rho(E)$ at low energies which generated the $\phi^{2} \ln \phi$ term. Initial numerical results indicate that non-perturbative effects remain in the $O\left(\phi^{3}\right)$ contribution to the free energy. This would have interesting implications for behavior near the phase transition.

Usually an $O\left(\phi^{3}\right)$ term in the free energy

$$
G=a t \phi^{2}+\gamma \phi^{3}+b \phi^{4}+\ldots,
$$

where $t \equiv T-T_{c}(H)$, signals a first order phase transition. Defining

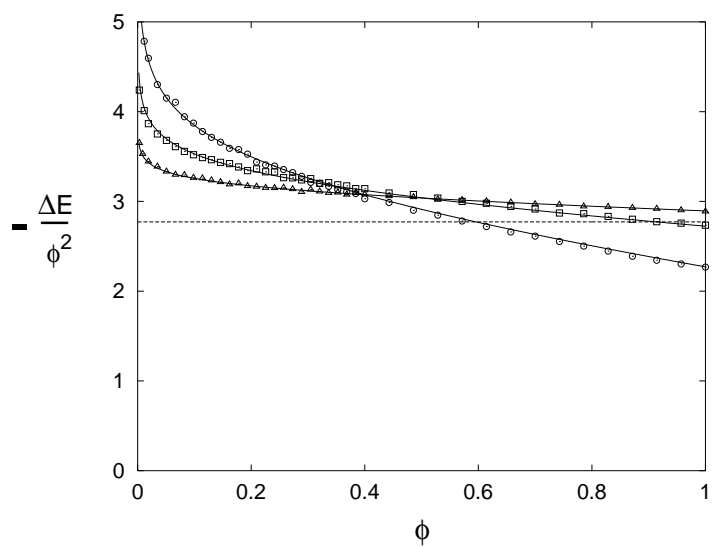

FIG. 3. Change in ground state energy vs. order parameter strength $\phi$ for the random matrix ensemble defined in the text. Horizontal dashed line is the perturbative $N \rightarrow \infty$ result. Points are for $N=5,10,20,(\circ, \square, \Delta)$ and solid lines are fits to the form of Eq. (12): $\Delta E / \phi^{2}=\alpha \log \phi-\beta+\gamma \phi$. Both $\alpha$ and $\gamma$ decrease as $\sim 1 / N$. $10^{4}$ matrices were used for each point. 


$$
\phi^{*} \equiv \gamma / b \quad, \quad t^{*} \equiv 9 \gamma^{2} / 32 a b,
$$

and keeping terms up to $O\left(\phi^{4}\right)$, we have that a second minimum appears in $G(\phi)$ for temperatures below $t^{*}$ and becomes energetically favorable compared to the $\phi=0$ solution when $t<t_{1}=(8 / 9) t^{*}$. More precisely, the two non-zero extrema of $G$ which occur at temperatures below $t^{*}$ are at

$$
\phi_{ \pm}=\frac{3}{8} \phi^{*}\left(-1 \pm \sqrt{1-\left(t / t^{*}\right)}\right)
$$

For negative $\gamma, \phi_{+}$is the local minimum which becomes energetically favorable below $t_{1}$, causing a first-order transition. For positive $\gamma$, the local minimum $\phi_{-}<0$. $\phi$, however, is the magnitude of the order parameter and must be positive, so this solution is not allowed. What happens instead, if $\gamma$ is positive, is that there is a continuous transition at $t=0$ as the $\phi_{+}$solution becomes an allowed minimum. Near this transition, for $|t| \ll t^{*}$, expanding the square root yields

$$
\phi \approx \frac{3 \phi^{*}}{16 t^{*}}|t|
$$

The scale for this deviation from the familiar mean-field behavior $\phi \propto \sqrt{|t|}$ is set by $t^{*}$ : for $|t| \gg t^{*}$ the squareroot behavior is recovered. The same behavior occurs if the field is varied at fixed temperature; $t$ is replaced by $h \equiv H-H_{c 2}(T)$ and the result is $\phi \propto|h|$ for $|h| \ll h^{*}$. Although the models considered here give a negative $\gamma$, favoring the continuous transition, the possibility that additional physics enters at sufficiently small $\phi$, and generates a positive $\gamma$, can not be ruled out.

The deviations from standard mean field behavior described here would be difficult to see in resistivity measurements, the most common measurements done near $H_{c 2}$ on conventional materials. They would be more likely to be seen in sensitive heat capacity, magnetization, or tunneling measurements. The form Eq. (13) for the free energy gives $G \propto\left(T-T_{c}\right)^{3}$ near the continuous transition (for $t \ll t^{*}$ ), instead of the Ginzburg-Landau behavior $G \propto\left(T-T_{c}\right)^{2}$, and leads to a kink in the heat capacity rather than an abrupt discontinuity. The magnetization $M \propto \phi^{2}$ would vary as $\left(T_{c}-T\right)^{2}$ rather than $\left(T_{c}-T\right)$. In the perturbative mean field theory, the spectrum near $H_{c 2}$ changes by $O\left(\phi^{2}\right)$. Non-perturbative effects arise from states close to the Fermi energy being strongly affected by the pairing even as $\phi \rightarrow 0$. This leads to a zero-bias anomaly in the spectrum: a peak near the Fermi energy, a $\phi^{2} / E^{2}$ rise, at the center of which is is a sharp spike giving $\rho\left(E_{F}\right)=0$.

Non-perturbative effects appear at scales much larger than the deviations from mean field behavior expected from phase fluctuations. The phase fluctuations which cause vortex lattice melting are believed to become important on the scale of critical phenomena, as estimated by the Ginzburg criterion [11]. This scale is
$\left(T_{G}-T_{c}\right) / T_{c} \lesssim 10^{-16}$ in zero magnetic field and $\lesssim 10^{-7}$ at $H=10 T$ for typical parameters of bulk conventional superconductors, although the scale may be much larger in high- $T_{c}$ materials 11]. The scale for the deviations described here is $1 / \sqrt{N_{f}}$ which can be $1 \%-10 \%$ for conventional supercondcutors.

I would like to thank R. B. Laughlin for support from NSF Grant No. DMR-9120361. This work was also supported by a Miller Research Fellowship from the Miller Institute for Basic Research in Science.

[1] L. P. Gor'kov, Zh. Eksperim. i Teor. Fiz. 37, 833 (1959); translated in Soviet Phys. JETP 10, 593 (1960).

[2] Reviewed in A. L. Fetter and P. C. Hohenberg in Superconductivity, edited by R. D. Parks (Marcel Dekker, New York, 1969).

[3] L. W. Gruenberg and L. Gunther, Phys. Rev. 176, 606 (1968).

[4] M. R. Norman, A. H. MacDonald, and H. Akera, Phys. Rev. B 51, 5927 (1995); T. Maniv, A. I. Rom, I. D. Vagner, and P. Wyder, Phys. Rev. B 46, 8360 (1992).

[5] Analytical results for the "quantum regime" of pairing within one Landau level (reviewed in M. Rasolt and Z. Tesanovic, Rev. Mod. Phys. 64, 709 (1992)) require fields $H>10^{3} T$ for conventional superconductors, which leads to certain difficulties (see C. T. Rieck et al., Physica C 170, 195 (1990)). This is not the regime discussed here: we consider effects near the conventional $H_{c 2}$ in which many Landau levels are being paired.

[6] S. R. Bahcall, Phys. Rev. Lett. 75, 1376 (1995).

[7] R. B. Laughlin, Phys. Rev. B 23, 5632 (1981).

[8] B. I. Halperin, Phys. Rev. B 25, 2185 (1982).

[9] See H. M. Jaeger, D. B. Havliand, B. G. Orr, and A. M. Goldman, Phys. Rev. B 40182 (1989) and references therein.

[10] P. Fulde and R. A. Ferrell, Phys. Rev. 135, 550 (1964).

[11] See for example C. J. Lobb, Phys. Rev. B 36, 3930 (1987), or G. Blatter et al., Rev. Mod. Phys. 66, 1124 (1994). 